\section{The Use of SEM and Other Complimentary Techniques for the Determination of Properties of Cementitious Materials}

\author{
Eric A. Draper \& Jan Skalny \\ RJ Lee Group, Inc., Monroeville, PA 15146
}

The need for continued rehabilitation of our concrete infrastructure has lead to the adaptation of modern "state-of-the-art" analytical methods for the characterization of concrete and other cementitious materials. Some of these techniques have not, until relatively recently, been commonly associated with the evaluation of concrete but are very useful both as tools for quality assurance and in the determination of the extent of existing damage. The technique of interest here is the coordinated electron-optical microscopic evaluation of concrete

Concrete is the most widely used building material in the world. Contrary to popular belief, concrete is not inert but chemically very complex and dynamic. While it is true that, pound for pound, concrete and its raw materials (cement, aggregate and water) are the most inexpensive building materials available for construction, it is also true that it responds to its environment in numerous and sometimes very subtle ways. These responses may sometimes result in a loss of durability and tremendous amounts of time and money being expended while searching for the cause(s) of the problem and providing a cost-effect solution. A quick survey of any large metropolitan area and the on-going construction repairs to highways and bridge decks there will quickly confirm this.

Traditional bulk analytical methods for testing concrete typically yield information on the performance of the concrete as a whole. That is to say that information on specific components of the concrete (cement, sand, coarse aggregate, etc.) and their interactions, may not be available. This is especially true when examining concrete for a potentially harmful reaction between the aggregate and cement paste (the product of mixing cement powder and water), known as alkali-silica reaction (ASR). Briefly stated, this reaction involves the production of an expansive gel (called ASR gel) within the concrete structure which can, subsequent to its formation, cause the structure to crack and require replacement. Petrographic methods for concrete exainination according to ASTM are also typically used in cases such as these, but positive identification of the ASR gel, which is essential here, may not be possible using optical methods alone. This is especially tue if the cracks in the aggregate and cement paste are very fine and only minor amounts of gel are present. Since the chemical composition of the gel can also vary, within limits, it becomes very important to know what this composition is. SEM, microprobe and X-ray analyses are of use in this case, but it is typical, because of different sample prep requirements, to utilize different samples for the various analytical techniques. This can create a problem when trying to correlate the information obtained from each analytical method. One of the greatest problems when analyzing the same sample using different techniques has always been relocating identical areas of interest so that a direct comparison of the analytical results is possible. This process has typically been accomplished by painting or scribing marks on the sample in the area of interest, then searching with the second instrument for the marks so that the analysis may be performed. The recent development of computer-controlled optical and electron microscopes and their stages makes this procedure less time consuming and more accurate. Computer-controlled stages, along with the software to efficiently run them, have been developed to permit relocation of identical areas observed in the stereo optical or petrographic microscopes, and in the SEM or vice versa. In addition, digital imaging and database techniques have been developed which permit the images to be stored or retrieved from any of the instruments.

As an illustration of the effectiveness of this complimentary technique, a section of concrete was prepared and analyzed. Figure 1 is a stereo optical image of a polished section showing a cracked aggregate in cement paste and what appears to be some reaction product (ASR gel?) within the cracks. Since the aggregate type directly influences the formation mechanism and occurrence of ASR gel, the mineralogical composition of the aggregate as well as the composition of the material within the cracks must be determined. Coordinates and images of these features were ubtained from the sample and stored. Figures 2 is a BSE image of the area in question at $10 \mathrm{X}$ magnification. Figure 3 is an $\mathrm{X}$-ray map of the same area showing the elemental distribution of the area in question and indicating that the cracked aggregate is quart. It also indicates the presence of ASR gel in the crack. Figure 4 shows the $X$-ray spectum obtained from the gel within the cracks and serves as additional confimation of the identification.

Because of our dependence on concrete as an infrastructure material, we need to employ all the means at our disposal in order to understand how it responds to its environment. This will enable us to more accurately predict how it will perform during its service life. Supplied with this khowledge, we can then design concrete structures for optimum durability. Methods and analytical techniques traditionally developed for materials analysis can be used with excellent results when evaluating concrete, especially when used in conjunction with each other. Computer programs and computer-controlled equipment designed to maximize and compliment each instruments capabilities are essential for the efficient acquisition of the data.
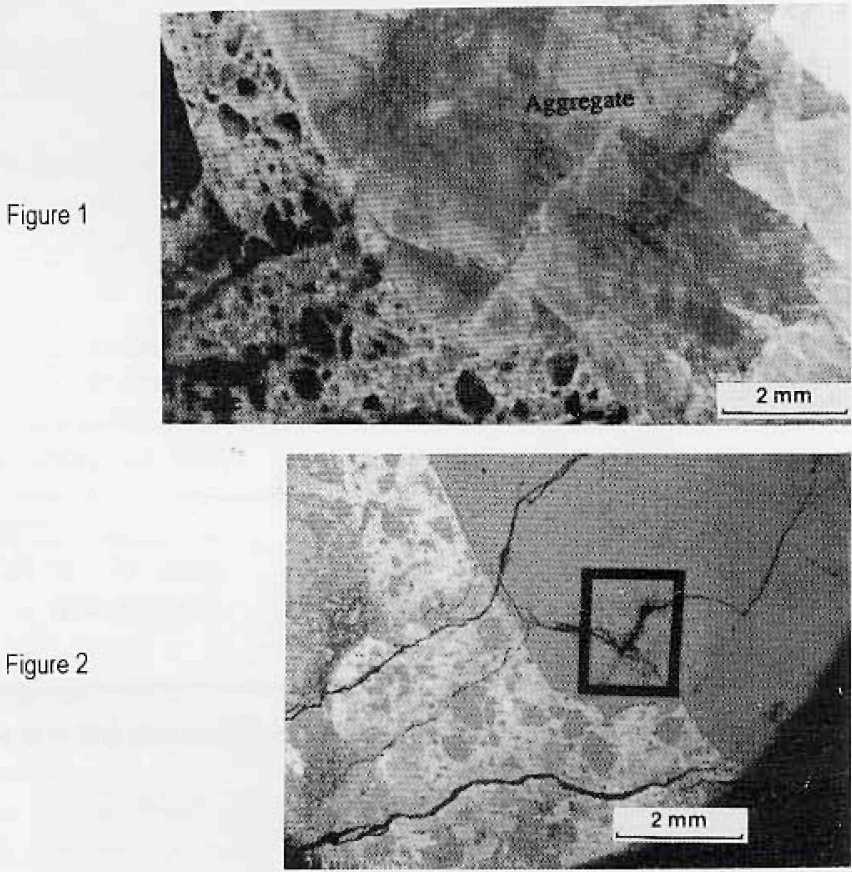

Figure 2

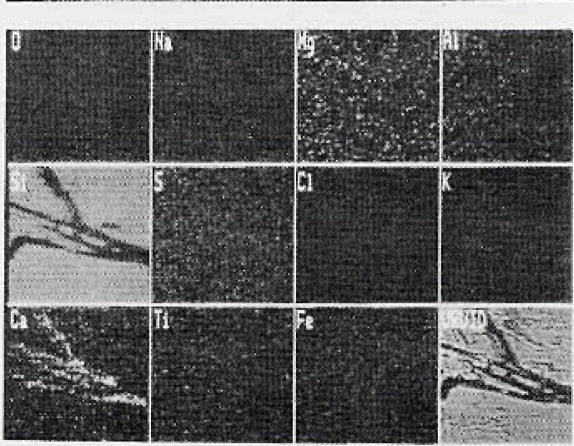

Figure 3

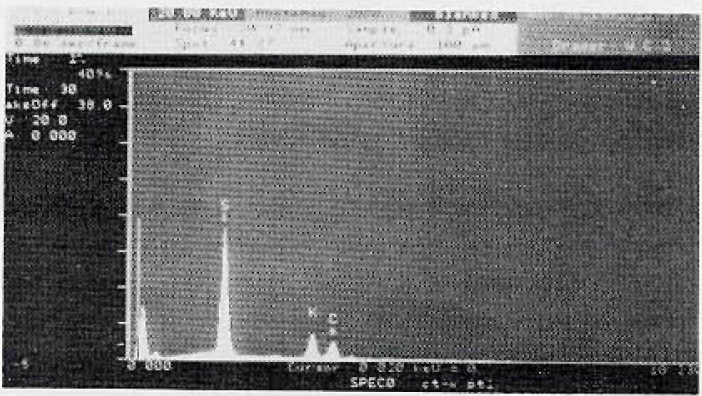

